\title{
A nomogram for predicting mortality in patients with COVID-19 and solid tumors: a multicenter retrospective cohort study
}

\author{
Chao Liu, ${ }^{1,2} \mathrm{Li} \mathrm{Li},{ }^{3}$ Kehan Song, ${ }^{4}$ Zhi-Ying Zhan, ${ }^{3}$ Yi Yao, ${ }^{1}$ Hongyun Gong, ${ }^{1}$ \\ Yuan Chen, ${ }^{5}$ Qun Wang, ${ }^{6}$ Xiaorong Dong, ${ }^{7}$ Zhibin Xie, ${ }^{8}$ Chun-Quan Ou (D) , 3 \\ Qinyong Hu, ${ }^{1}$ Qibin Song ${ }^{1}$
}

To cite: Liu C, Li L, Song K, et al. A nomogram for predicting mortality in patients with COVID-19 and solid tumors: a multicenter retrospective cohort study. Journal for ImmunoTherapy of Cancer 2020;8:e001314. doi:10.1136/ jitc-2020-001314

- Additional material is published online only. To view please visit the journal online (http://dx.doi.org/10.1136/jitc2020-001314).

$\mathrm{CL}, \mathrm{LL}$ and $\mathrm{KS}$ contributed equally.

Accepted 27 August 2020

Check for updates

(C) Author(s) (or their employer(s)) 2020. Re-use permitted under CC BY-NC. No commercial re-use. See rights and permissions. Published by BMJ.

For numbered affiliations see end of article.

\section{Correspondence to}

Professor Chun-Quan Ou; ouchunquan@hotmail.com

Professor Qinyong Hu; rm001223@whu.edu.cn

Professor Qibin Song; qibinsong@whu.edu.cn

\section{ABSTRACT}

Background Individualized prediction of mortality risk can inform the treatment strategy for patients with COVID-19 and solid tumors and potentially improve patient outcomes. We aimed to develop a nomogram for predicting in-hospital mortality of patients with COVID-19 with solid tumors.

Methods We enrolled patients with COVID-19 with solid tumors admitted to 32 hospitals in China between December 17, 2020, and March 18, 2020. A multivariate logistic regression model was constructed via stepwise regression analysis, and a nomogram was subsequently developed based on the fitted multivariate logistic regression model. Discrimination and calibration of the nomogram were evaluated by estimating the area under the receiver operator characteristic curve (AUC) for the model and by bootstrap resampling, a Hosmer-Lemeshow test, and visual inspection of the calibration curve. Results There were 216 patients with COVID-19 with solid tumors included in the present study, of whom 37 (17\%) died and the other 179 all recovered from COVID-19 and were discharged. The median age of the enrolled patients was 63.0 years and $113(52.3 \%)$ were men. Multivariate logistic regression revealed that increasing age $(0 R=1.08,95 \% \mathrm{Cl} 1.00$ to 1.16), receipt of antitumor treatment within 3 months before COVID-19 (OR=28.65, $95 \% \mathrm{Cl} 3.54$ to 231.97$)$, peripheral white blood cell (WBC) count $\geq 6.93 \times 10^{9} / \mathrm{L}(\mathrm{OR}=14.52,95 \% \mathrm{Cl} 2.45$ to 86.14), derived neutrophil-to-lymphocyte ratio (dNLR; neutrophil count/(WBC count minus neutrophil count)) $\geq 4.19$ (OR=18.99, 95\% Cl 3.58 to 100.65), and dyspnea on admission ( $\mathrm{OR}=20.38,95 \% \mathrm{Cl} 3.55$ to 117.02$)$ were associated with elevated mortality risk. The performance of the established nomogram was satisfactory, with an AUC of 0.953 (95\% Cl 0.908 to 0.997$)$ for the model, non-significant findings on the Hosmer-Lemeshow test, and rough agreement between predicted and observed probabilities as suggested in calibration curves. The sensitivity and specificity of the model were $86.4 \%$ and $92.5 \%$.

Conclusion Increasing age, receipt of antitumor treatment within 3 months before COVID-19 diagnosis, elevated WBC count and dNLR, and having dyspnea on admission were independent risk factors for mortality among patients with COVID-19 and solid tumors. The nomogram based on these factors accurately predicted mortality risk for individual patients.

\section{INTRODUCTION}

COVID-19 poses an unprecedented threat to global health, with the WHO officially denoting the outbreak as a 'public health emergency of international concern' on March 11, 2020. ${ }^{1}$ As of 10 AM CEST on June $19,2020,8,385,440$ individuals had been confirmed to be infected by SARS-CoV-2, and 450,686 people had died of COVID-19. ${ }^{2}$ Cancer was newly diagnosed in 18.1 million people and 9.6 million people died of cancer worldwide in 2018. ${ }^{34}$ Patients with cancer are more vulnerable to SARS-CoV-2 infection given the relatively high prevalence of underlying chronic disease and cancer-induced and anticancer therapy-induced systemic immunosuppressive states. ${ }^{5}$ Patients with cancer who are also infected with SARS-CoV-2 may be at higher risk of severe events and deteriorate more rapidly than patients without cancer. ${ }^{7-9}$ Predicting the prognosis for patients with COVID-19 and cancer can help in prioritizing patient care resources and informing the choice of treatment strategy, which presumably would improve patient outcomes.

SARS-CoV-2 infection can deregulate immune responses, which may trigger viral hyperinflammation in patients with severe COVID-19. ${ }^{10}$ Moreover, an uncontrolled and overwhelming systemic inflammatory response can result in acute respiratory distress syndrome (ARDS), which is the principal cause of death among patients with COVID$19 .^{11}$ Logically, then, indicators of inflammation could be useful for predicting prognosis in patients with COVID-19. Several important inflammation-related indices derived from routine blood tests are generally available for 
patients with COVID-19 on admission. Hence, applying statistical modeling to inflammation-related indices may be an economic and effective way to predict disease outcomes.

The prognosis for patients with COVID-19 and cancer could also be associated with cancer characteristics and symptoms on admission. Although mortality is the endpoint of greatest concern in both cancer and COVID19 , little information is available on the possible relevance of cancer characteristics and symptoms on admission in predicting mortality for patients with COVID-19. Only a few studies on a small numbers of patients with cancer focused on the outcome of composite severe events including admission to an intensive care unit, the use of mechanical ventilation, or death, since most patients were still hospitalized at the time of reporting the result of studies. A previous study of 28 patients indicated that patients with COVID-19 and solid cancer who underwent antitumor treatment within 14 days of the COVID-19 diagnosis were at greater risk of severe events than those who did not receive treatment during that interval ${ }^{12}$; also, a national analysis of 1590 patients with COVID-19, among whom 18 had a history of cancer, revealed that patients receiving surgery or chemotherapy within 1 month of the COVID-19 diagnosis had a higher risk of severe events. ${ }^{9}$ Only one study reported a higher risk of death among those receiving chemotherapy within 4 weeks before symptom onset. ${ }^{13}$ Elevated immunosuppression or dysfunction could explain the increase in risk of severe events among patients with COVID-19 and cancer who received antitumor treatment. Likewise, inflammationrelated indices may indicate prognosis for patients with COVID-19 with cancer as well. However, to the best of our knowledge, whether inflammation-related indices can be used to predict mortality risk among SARS-CoV-2-infected patients with cancer has not been reported.

Nomograms have been used extensively predicting prognosis in cancer, mainly because they offer a userfriendly graphic presentation of the estimated probabilities of an event, such as death, that is tailored to individual patients. ${ }^{14}$ Here, we sought to identify prognostic factors for patients with COVID-19 and solid tumors and to develop a nomogram for predicting mortality risk in a multicenter retrospective cohort study of 216 patients with COVID-19 and solid tumors in China.

\section{PATIENTS AND METHODS \\ Subjects}

In this retrospective cohort study, subjects were consecutive patients with solid tumors who had been diagnosed with COVID-19 and admitted to 32 hospitals in China from December 17, 2020, to March 18, 2020. Inclusion criteria were: (1) pathologically confirmed solid tumor; (2) confirmed diagnosis of COVID-19 by detection of SARS-CoV-2 via real-time PCR; (3) age $>18$ years old. Patients with benign tumors were excluded.

\section{Data collection}

Information on patient demographics (age and sex), smoking history (yes/no), cancer characteristics (type, stage, receipt of antitumor treatment within 3 months before COVID-19 diagnosis), comorbid conditions, signs and symptoms, laboratory and radiology findings, complications, receipt of steroid, and clinical outcomes was extracted from medical records by two physicians at each hospital independently. We considered several inflammation-related indices derived from routine blood tests, including peripheral white blood cell (WBC) count, absolute lymphocyte count, absolute neutrophil count, neutrophil-to-lymphocyte ratio (NLR), and derived NLR (dNLR; neutrophil count/(WBC count minus neutrophil count)). Cancer types included lung, breast, gastrointestinal, gynecologic, head and neck, and urogenital. Antitumor treatments included surgery, chemotherapy, radiotherapy, targeted therapy, immunotherapy, endocrine therapy, and combination therapy. The follow-up period ended on May 17, 2020, when outcomes for all patients had been clearly identified. Cause of death for each patient was determined by both physicians and oncologists. All discharged patients met the following criteria: (1) body temperature had returned to normal for more than 3 days; (2) respiratory symptoms had improved notably; (3) lung imaging findings indicated significant alleviation of acute exudative lesions in the lung; and (4) nasal and pharyngeal swab samples were negative for the presence of SARS-CoV-2 on two consecutive occasions at least 24 hours apart. ${ }^{15}$

\section{Statistical analysis}

The included patients were classified into two groups according to clinical outcomes: non-survivors and survivors. Continuous variables were described as medians and IQRs, and categorical variables as counts and percentages. The Mann-Whitney U test was applied to compare continuous variables and cancer stage between two groups, and Fisher's exact test was used to compare categorical variables between groups.

We developed a nomogram for predicting in-hospital mortality among patients with COVID-19 and solid tumors in four steps: (1) fitting a univariate logistic regression model with one variable at a time (ie, demographics, smoking history, cancer characteristics, comorbidities, symptoms, and radiologic findings on admission); (2) determining the cutoff values for inflammation-related indices by using receiver operator characteristic (ROC) curve analysis, classifying the inflammation-related indices into high and low levels according to the optimal cutoff values, and then fitting the univariate logistic regression model with one dummy variable of inflammation-related index at a time; (3) performing multivariate logistic regression analysis with the inclusion of variables that were potential independent risk factors for mortality; (4) constructing the nomogram based on the multivariate logistic regression model. 
Additional details for step (3) are as follows. Age, sex, and any on-admission variables for which the $95 \%$ CI of the OR did not include 1 in univariate logistic regression analyses were candidate independent variables for the multivariate logistic regression analysis. To avoid multicollinearity, which could lead to instability in the multivariate logistic regression model, we first calculated the generalized variance inflation factor (GVIF) for each candidate independent variable and then removed the variable with the largest GVIF ${ }^{[1 /(2 \times d f)]}$ (where $d f$ is the degree of freedom associated with the term for a variable; $d s$ for variables other than cancer type were 1 in this study) at each step until the GVIF ${ }^{[1 /(2 \times d f]}$ for all remaining variables were less than 2.24 (ie, $\left.5^{[1 / 2]}\right) .{ }^{16}$ Subsequently, we fitted a full model with the remaining variables and conducted further stepwise regression with a backward selection procedure to obtain a simplified model that corresponded to the smallest Akaike information criterion. ${ }^{17}$

We evaluated the performance of the nomogram in terms of discrimination and calibration. ${ }^{14}{ }^{18}$ Specifically, we assessed its ability to distinguish non-survivors from survivors by estimating the area under the ROC curve (AUC) by using the method developed by DeLong et al. ${ }^{19}$ In addition, the predictive performance of the model was validated with bootstrap resampling repeated 1000 times. Calibration was examined by using a Hosmer-Lemeshow test and by comparing the predicted and observed probabilities of mortality in patients with COVID-19 with solid tumors. ${ }^{14} 1820$ We conducted sensitivity analyses in which (1) cancer type was classified into lung cancer and other cancers; (2) multivariate logistic regression models were fitted with additional one variable of sign on admission. Missing data were not imputed in this study. $p<0.05$ was considered statistically significant. R software V.3.6.2 (R Foundation for Statistical Computing) was used for all analyses.

\section{RESULTS}

Figure 1 illustrates the flow of selection of patients in the current study. A total of 216 patients were included. At the time of analysis, 37 patients $(17 \%)$ had died and 179 patients (83\%) had recovered from COVID-19 and had been discharged from the hospital. The age of the 216 patients ranged from 22 to 91 years (median 63.0 years); slightly more than half $(113,52.3 \%)$ were men. Patients with lung cancer represented the largest proportion of non-survivors $(37.8 \%)$, with only $19.6 \%$ of survivors having this type of cancer. Proportions of patients with gastrointestinal cancer were high both among nonsurvivors $(32.4 \%)$ and survivors $(31.3 \%)$. Half of patients who did not survive had stage IV cancer, and survivors were evenly distributed by cancer stage. More patients who had died had received antitumor treatment within 3 months (65.4\%) and steroid (62.2\%) than did patients who survived $(38.1 \% ; 35.2 \%)$. Heart rate was on average faster among patients who had died $(96.0 \mathrm{bpm})$ than

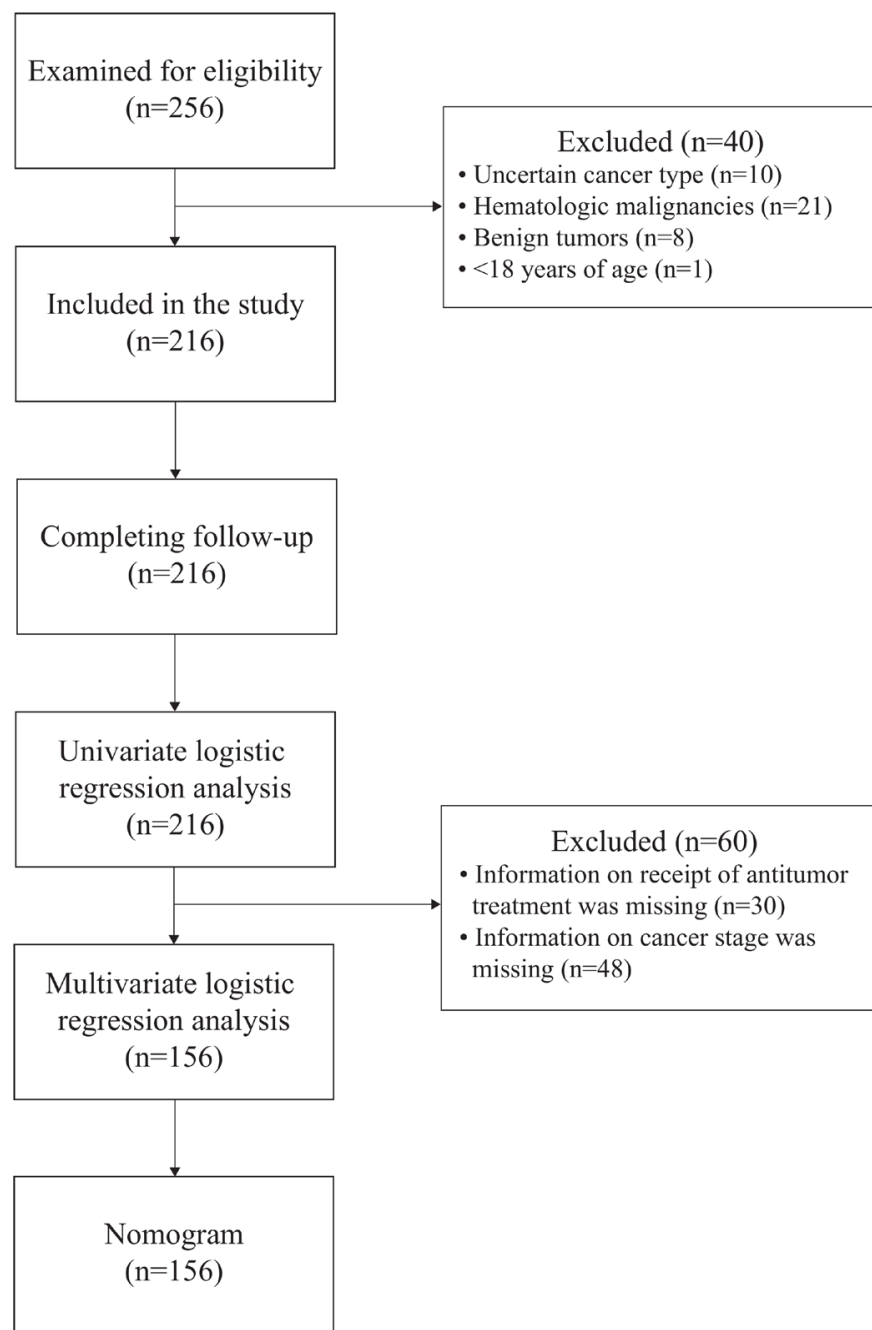

Figure 1 Flow diagram of selection of patients.

among those who survived $(85.0 \mathrm{bpm})$. Chronic obstructive pulmonary disease was more common among nonsurvivors than among survivors $(21.6 \%$ vs $7.3 \%)$, and dyspnea and fatigue were more common among nonsurvivors as well. Complications of stroke, ARDS, acute heart failure, acute renal failure, and arrhythmia were also more common among non-survivors. No statistically significant differences were found in other factors between the two groups (table 1 ).

In terms of laboratory findings, median WBC (7.5 vs $\left.5.0 \times 10^{9} / \mathrm{L}\right)$, neutrophils $\left(6.6\right.$ vs $\left.3.4 \times 10^{9} / \mathrm{L}\right)$, NLR ( 10.9 vs 3.4 ), and dNLR (6.1 vs 2.2) were all higher among nonsurvivors than survivors. Conversely, lower lymphocyte counts $\left(0.5\right.$ vs $\left.1.0 \times 10^{9} / \mathrm{L}\right)$ were observed among the nonsurvivors (table 1). The optimal cutoff points for these variables identified by ROC analysis were $6.93 \times 10^{9} / \mathrm{L}$ for WBC count, $0.70 \times 10^{9} / \mathrm{L}$ for lymphocyte count, $5.82 \times 10^{9} / \mathrm{L}$ for neutrophil count, 7.05 for NLR, and 4.19 for dNLR. The AUCs for neutrophil count (0.79), NLR (0.85), and dNLR (0.85) were larger than those for WBC count $(0.74)$, with sensitivity of $65 \%-78 \%$ and specificity of $80 \%-88 \%$ (figure 2 , table 2 ). 
Table 1 Patient, disease, and treatment variables at admission for patients with COVID-19 and solid tumors

\begin{tabular}{|c|c|c|c|c|c|}
\hline \multirow[b]{2}{*}{ Characteristics } & \multirow{2}{*}{$\begin{array}{l}\text { All patients } \\
(n=216)\end{array}$} & \multicolumn{2}{|l|}{ Groups } & \multirow[b]{2}{*}{$P$ value } & \multirow[b]{2}{*}{ OR $(95 \% \mathrm{Cl})^{*}$} \\
\hline & & Non-survivors $(n=37)$ & Survivors $(n=179)$ & & \\
\hline \multicolumn{6}{|l|}{ Demographics } \\
\hline Sex, male, frequency (\%) & $113 / 216(52.3)$ & $25 / 37(67.6)$ & $88 / 179(49.2)$ & 0.048 & 2.15 (1.02 to 4.55$)$ \\
\hline $\begin{array}{l}\text { Smoking history, frequency } \\
(\%)\end{array}$ & $58 / 216(26.9)$ & $14 / 37(37.8)$ & $44 / 179(24.6)$ & 0.106 & 1.87 (0.89 to 3.94$)$ \\
\hline Breast cancer & $34 / 216(15.7)$ & $1 / 37(2.7)$ & $33 / 179(18.4)$ & - & Ref \\
\hline Gynecological cancer & $17 / 216(7.9)$ & $1 / 37(2.7)$ & $16 / 179(8.9)$ & - & Ref \\
\hline Head and neck cancer & $26 / 216(12.0)$ & $1 / 37(2.7)$ & $25 / 179(14.0)$ & - & Ref \\
\hline Gastrointestinal cancer & $68 / 216(31.5)$ & $12 / 37(32.4)$ & $56 / 179(31.3)$ & - & 5.29 (1.42 to 19.61$)$ \\
\hline I & $43 / 168(25.6)$ & $2 / 26(7.7)$ & $41 / 142$ (28.9) & - & - \\
\hline II & 40/168 (23.8) & $7 / 26(26.9)$ & 33/142 (23.2) & - & - \\
\hline III & $37 / 168$ (22.0) & $4 / 26(15.4)$ & $33 / 142(23.2)$ & - & - \\
\hline IV & 48/168 (28.6) & $13 / 26(50.0)$ & $35 / 142(24.6)$ & - & - \\
\hline $\begin{array}{l}\text { Receipt of antitumor } \\
\text { treatment, frequency (\%) }\end{array}$ & $78 / 186(41.9)$ & $17 / 26(65.4)$ & $61 / 160(38.1)$ & 0.011 & 3.06 (1.29 to 7.30$)$ \\
\hline WBC count, $\times 10^{9} / \mathrm{L}$ & $5.3(4.1-7.1)$ & $7.5(5.7-10.4)$ & $5.0(4.0-6.4)$ & $<0.001$ & - \\
\hline Lymphocyte count, $\times 10^{9} / \mathrm{L}$ & $0.9(0.6-1.4)$ & $0.5(0.4-0.8)$ & $1.0(0.7-1.5)$ & $<0.001$ & - \\
\hline Neutrophil count, $\times 10^{9} / \mathrm{L}$ & $3.8(2.6-5.5)$ & $6.6(4.5-8.9)$ & $3.4(2.5-4.7)$ & $<0.001$ & - \\
\hline NLR & $4.0(2.3-8.0)$ & $10.9(7.2-19.5)$ & $3.4(2.1-6.3)$ & $<0.001$ & - \\
\hline COPD & $21 / 216(9.7)$ & $8 / 37(21.6)$ & $13 / 179(7.3)$ & 0.013 & 3.52 (1.34 to 9.24$)$ \\
\hline Chronic liver & $13 / 216(6.0)$ & $3 / 37(8.1)$ & $10 / 179(5.6)$ & 0.471 & $1.49(0.39$ to 5.71$)$ \\
\hline Chronic renal & $9 / 216(4.2)$ & $1 / 37(2.7)$ & $8 / 179(4.5)$ & 1.000 & 0.59 (0.07 to 4.89$)$ \\
\hline \multicolumn{6}{|l|}{ Signs, median (IQR) } \\
\hline Heart rate, bpm & $86.0(77.0-96.5)$ & $96.0(77.0-104.0)$ & $85.0(77.0-95.0)$ & 0.038 & - \\
\hline $\begin{array}{l}\text { Systolic blood pressure, } \\
\mathrm{mm} \mathrm{Hg}\end{array}$ & $128.0(120.0-140.0)$ & $125.0(115.0-141.0)$ & $128.0(120.0-139.5)$ & 0.493 & - \\
\hline $\begin{array}{l}\text { Diastolic blood pressure, } \\
\mathrm{mm} \mathrm{Hg}\end{array}$ & $78.5(70.8-86.0)$ & $79.0(71.0-87.0)$ & $78.0(70.5-86.0)$ & 0.642 & - \\
\hline $\begin{array}{l}\text { Respiratory rate, breaths per } \\
\text { minute }\end{array}$ & $20.0(20.0-22.0)$ & $20.0(20.0-23.0)$ & $20.0(19.0-22.0)$ & 0.039 & - \\
\hline Temperature, ${ }^{\circ} \mathrm{C}$ & $36.7(36.5-37.5)$ & $36.8(36.5-38.0)$ & $36.7(36.5-37.5)$ & 0.472 & - \\
\hline \multicolumn{6}{|l|}{ Symptoms, frequency (\%) } \\
\hline Fever & $160 / 216(74.1)$ & 26/37 (70.3) & $134 / 179(74.9)$ & 0.543 & 0.79 (0.36 to 1.74$)$ \\
\hline Cough & $141 / 216(65.3)$ & $24 / 37$ (64.9) & $117 / 179(65.4)$ & 1.000 & 0.98 (0.47 to 2.05$)$ \\
\hline Sputum & $88 / 216(40.7)$ & 18/37 (48.6) & 70/179 (39.1) & 0.358 & $1.48(0.72$ to 3.01$)$ \\
\hline Dyspnea & $85 / 216(39.4)$ & 26/37 (70.3) & $59 / 179$ (33.0) & $<0.001$ & 4.81 (2.22 to 10.38$)$ \\
\hline Fatigue & 108/216 (50.0) & $25 / 37(67.6)$ & $83 / 179(46.4)$ & 0.029 & 2.41 (1.14 to 5.09 ) \\
\hline Headache & $17 / 216$ (7.9) & $5 / 37(13.5)$ & $12 / 179(6.7)$ & 0.179 & $2.17(0.72$ to 6.60$)$ \\
\hline
\end{tabular}


Table 1 Continued

\begin{tabular}{|c|c|c|c|c|c|}
\hline \multirow[b]{2}{*}{ Characteristics } & \multirow{2}{*}{$\begin{array}{l}\text { All patients } \\
(n=216) \\
\end{array}$} & \multicolumn{2}{|l|}{ Groups } & \multirow[b]{2}{*}{$P$ value } & \multirow[b]{2}{*}{ OR $(95 \% \mathrm{Cl})^{*}$} \\
\hline & & Non-survivors $(n=37)$ & Survivors $(n=179)$ & & \\
\hline Muscle ache & 28/216 (13.0) & 2/37 (5.4) & $26 / 179(14.5)$ & 0.181 & 0.34 (0.08 to 1.48$)$ \\
\hline Diarrhea & 29/216 (13.4) & 4/37 (10.8) & 25/179 (14.0) & 0.793 & 0.75 (0.24 to 2.29$)$ \\
\hline Nausea & $19 / 216(8.8)$ & 2/37 (5.4) & $17 / 179(9.5)$ & 0.540 & 0.54 (0.12 to 2.46$)$ \\
\hline Anorexia & $10 / 216(4.6)$ & $1 / 37(2.7)$ & $9 / 179(5.0)$ & 1.000 & 0.52 (0.06 to 4.27$)$ \\
\hline $\begin{array}{l}\text { Radiologic findings, frequency } \\
(\%)\end{array}$ & & & & 0.459 & \\
\hline No involvement & 6/195 (3.1) & $0(0.0)$ & 6/167 (3.6) & - & Ref \\
\hline \multicolumn{6}{|l|}{ Complications, frequency (\%) } \\
\hline Stroke & $30 / 213(14.1)$ & $30 / 35(85.7)$ & $0 / 178(0.0)$ & $<0.001$ & - \\
\hline ARDS & 62/213 (29.1) & $35 / 36(97.2)$ & 27/177 (15.3) & $<0.001$ & - \\
\hline Acute heart failure & $9 / 212(4.2)$ & 8/34 (23.5) & $1 / 178(0.6)$ & $<0.001$ & - \\
\hline Acute renal failure & 6/214 (2.8) & 6/36 (16.7) & $0 / 178(0.0)$ & $<0.001$ & - \\
\hline Pulmonary embolism & $1 / 213(0.5)$ & $0 / 35(0.0)$ & $1 / 178(0.6)$ & 1.000 & - \\
\hline Arrhythmia & $11 / 210(5.2)$ & 10/33 (30.3) & $1 / 177(0.6)$ & $<0.001$ & - \\
\hline
\end{tabular}

Continuous variables were summarized as median (IQR), and categorical variables as $\mathrm{n} / \mathrm{N}$ (\%), where $\mathrm{N}$ was the total number of patients without missing data. Continuous variables and cancer stage were compared between groups with Mann-Whitney $U$ tests, and categorical variables were compared with Fisher's exact tests. Sample size for analysis of cancer stage was 168; for antitumor treatment, 186; and for radiologic findings, 195. *We estimated ORs for potentially influential factors of mortality for patients with COVID-19 and solid tumors, including demographics, smoking history, cancer characteristics, comorbidities, symptoms, and radiologic findings on admission. ORs were provided for categorical variables of inflammation-related indices in table 2, but not for continuous variables of these indices.

†Breast cancer, gynecologic cancer, and head and neck cancer were treated as the reference group when estimating ORs for cancer type. ¥Cancer stage was treated as an ordinal variable when estimating OR.

ARDS, acute respiratory distress syndrome; COPD, chronic obstructive pulmonary disease; dNLR, derived NLR; NLR, neutrophil-to-lymphocyte ratio; $\mathrm{OR}$, odds ratio; WBC, peripheral white blood cell.

Age, sex, and the on-admission variables that were statistically significant in the univariate logistic regression analyses (ie, those for which the $95 \%$ CI of the OR did not include 1 (tables 1 and 2)) were considered for the multivariate logistic regression analysis. Neutrophil count was excluded since the inclusion of this variable led to serious multicollinearity in the multivariate model (online supplemental table S1). Finally, the backward stepwise regression (ie, simplified model) included six independent variables (ie, age, cancer stage, receipt of antitumor treatment, WBC count, dNLR, and dyspnea at admission). The final results of our simplified model revealed that the following five factors were statistically significantly associated with increased risk of mortality for patients with COVID-19 and solid tumors: age ( $\mathrm{OR}=1.08$, 95\% CI 1.00 to 1.16$)$, receiving antitumor treatment during the 3 months before COVID- 19 (OR=28.65, 95\% CI 3.54 to 231.97$)$, WBC count $\geq 6.93 \times 10^{9} / \mathrm{L}(\mathrm{OR}=14.52$, $95 \%$ CI 2.45 to 86.14$)$, dNLR $\geq 4.19$ (OR=18.99, 95\% CI
3.58 to 100.65$)$, presenting with dyspnea on admission $(\mathrm{OR}=20.38,95 \%$ CI 3.55 to 117.02$)$ (table 3$)$.

The nomogram for predicting the probability of death among patients with COVID-19 with solid tumors was constructed based on the multivariate logistic regression model. The nomogram had high discrimination, with an AUC of 0.953 (95\% CI 0.908 to 0.997 ) and good model sensitivity $(86.4 \%)$ and specificity $(92.5 \%)$. The optimismcorrected AUC obtained from bootstrap resampling was 0.934 , suggesting good internal validation (online supplemental figure S1). In addition, the Hosmer-Lemeshow test indicated that the model calibrated well $(\mathrm{p}=0.236)$, and the calibration curve showed that the predicted probabilities of mortality risk in general agreed well with the observed probabilities (online supplemental figure S2). In the nomogram, each value of a variable corresponds to a score, and the corresponding scores for the six variables included in the model are summed to achieve a total score for an individual. The total score is then projected onto a 


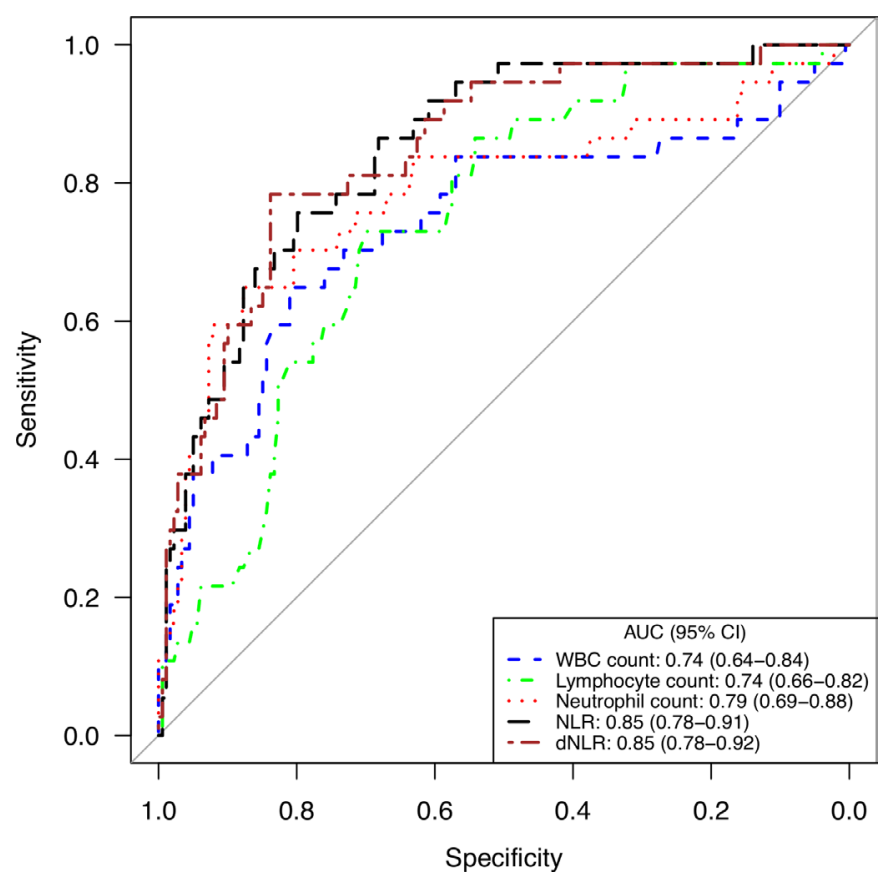

Figure 2 Receiver operator characteristic curves for inflammation-related indices for predicting mortality among patients with COVID-19 and solid tumors. AUC, the area under the receiver operator characteristic curve; dNLR, derived NLR; NLR, neutrophil-to-lymphocyte ratio; WBC, peripheral white blood cell.

total point scale to obtain the probability of mortality for an individual according to his or her profile (figure 3).

The results of univariate sensitivity analyses suggested that patients with lung cancer were at higher risk of death than those with other cancers in univariate logistic regression analysis ( $\mathrm{OR}=2.50,95 \% \mathrm{CI} 1.17$ to 5.36$)$. However, cancer type was excluded in the backward selection procedure and was not included in the multivariate logistic regression model. When adding heart rate and respiratory rate into the multivariate logistic regression models separately, consistent with the results of main analysis, elevated mortality risk was observed in patients with increasing age, receiving antitumor treatment within 3 months of COVID-19 diagnosis, with high WBC count and dNLR, and presenting with dyspnea on admission, while the associations between heart rate, respiratory rate and mortality were not statistically significant after adjustment of variables, which were included in the multivariate logistic regression model in the main analysis (online supplemental table S2).

\section{DISCUSSION}

We conducted a multicenter study of 216 patients with solid tumors and SARS-CoV-2 infection to identify risk factors for mortality, and we used the results to generate a nomogram involving patient age, cancer stage, receipt of antitumor treatment within 3 months before COVID-19 diagnosis, WBC count, dNLR, and the presence of dyspnea at admission to provide individualized predictions of mortality risk. Our nomogram performed well in terms of discrimination and calibration. To our knowledge, this is the first report of a quantitative nomogram for predicting mortality risk for patients with COVID-19 and solid tumors. These findings represent invaluable data on patients with COVID-19 and solid tumors obtained during the COVID-19 pandemic and have important implications for the clinical management of such cases. Patients at higher probability of death identified by the nomogram should be treated with extra care to prevent negative outcomes. Age, an important demographic variable, was included in the developed nomogram because we found that mortality risk increased with age; this finding agrees with that from a prior study from New York. ${ }^{21}$

Our nomogram also contained information on whether patients had received antitumor treatment within 3 months of the COVID-19 diagnosis, and our findings suggested that those who had were at higher risk of death than other patients. This finding is consistent with that of another study in which the crude mortality

Table 2 Comparisons of different inflammation-related indices in predicting mortality for patients with COVID-19 with solid tumors

\begin{tabular}{llllllr}
\hline $\begin{array}{l}\text { Inflammation-related } \\
\text { indices }\end{array}$ & AUC $(\mathbf{9 5 \%} \mathbf{C l})$ & P value* $^{*}$ & Thresholds & Sensitivity (\%) & Specificity (\%) & OR (95\% Cl) $\dagger$ \\
\hline WBC count, $\times 10^{9} / \mathrm{L}$ & $0.74(0.64$ to 0.84$)$ & - & 6.93 & 65 & 81 & $7.87(3.64$ to 17.03$)$ \\
\hline Lymphocyte count, $\times 10^{9} / \mathrm{L}$ & $0.74(0.66$ to 0.82$)$ & 0.992 & 0.70 & 73 & 70 & $6.25(2.83$ to 13.80$)$ \\
\hline Neutrophil count, $\times 10^{9} / \mathrm{L}$ & $0.79(0.69$ to 0.88$)$ & $<0.001$ & 5.82 & 65 & 88 & $13.17(5.86$ to 29.59$)$ \\
NLR & $0.85(0.78$ to 0.91$)$ & 0.015 & 7.05 & 76 & 80 & $12.35(5.36$ to 28.47$)$ \\
dNLR & $0.85(0.78$ to 0.92$)$ & 0.032 & 4.19 & 78 & 84 & $18.75(7.79$ to 45.11$)$ \\
\hline
\end{tabular}

${ }^{*}$ The receiver operator characteristic curves were compared by using the method developed by DeLong et al ${ }^{19}$ and WBC count was used as the reference predictor for the comparisons.

†ORs were estimated for comparing different levels of inflammation-related indices using the optimal cutoff values as the thresholds (WBC count $\geq 6.93 \mathrm{vs}<6.93 \times 10^{9} / \mathrm{L}$; lymphocyte count $\leq 0.70 \mathrm{vs}>0.70 \times 10^{9} / \mathrm{L}$; neutrophil count $\geq 5.82 \mathrm{vs}<5.82 \times 10^{9} / \mathrm{L}$; NLR $\geq 7.05 \mathrm{vs}$ $<7.05$; and dNLR $\geq 4.19$ vs $<4.19$ ).

AUC, the area under the receiver operator characteristic curve; dNLR, derived NLR; NLR, neutrophil-to-lymphocyte ratio; WBC, peripheral white blood cell. 
Table 3 Results of multivariate logistic regression models to assess predictors of mortality for patients with COVID-19 with solid tumors

\begin{tabular}{|c|c|c|c|c|}
\hline \multirow[b]{2}{*}{ Variables } & \multicolumn{2}{|l|}{ Full model } & \multicolumn{2}{|l|}{ Simplified model } \\
\hline & OR $(95 \% \mathrm{Cl})$ & $P$ value & OR $(95 \% \mathrm{Cl})$ & $P$ value \\
\hline Age, years & 1.09 (1.00 to 1.19$)$ & 0.060 & $1.08(1.00$ to 1.16$)$ & 0.043 \\
\hline \multicolumn{5}{|l|}{ Sex } \\
\hline Male vs female & 0.38 (0.06 to 2.32$)$ & 0.292 & - & \\
\hline \multicolumn{5}{|l|}{ Cancer type } \\
\hline Breast, gynecologic, head and neck cancer & Ref & - & - & - \\
\hline Gastrointestinal cancer & $5.84(0.43$ to 80.06$)$ & 0.187 & - & \\
\hline Lung cancer & 2.37 (0.21 to 27.33$)$ & 0.490 & - & - \\
\hline Urogenital cancer & 3.80 (0.20 to 72.67$)$ & 0.375 & - & - \\
\hline Cancer stage & 1.55 (0.70 to 3.45$)$ & 0.280 & $1.68(0.79$ to 3.56$)$ & 0.176 \\
\hline Receipt of antitumor treatment & 40.83 (4.14 to 403.20$)$ & 0.001 & 28.65 (3.54 to 231.97$)$ & 0.002 \\
\hline \multicolumn{5}{|l|}{ WBC count } \\
\hline$\geq 6.93 \mathrm{vs}<6.93 \times 10^{9} / \mathrm{L}$ & 27.19 (2.99 to 247.00$)$ & 0.003 & $14.52(2.45$ to 86.14$)$ & 0.003 \\
\hline \multicolumn{5}{|l|}{ Lymphocyte count } \\
\hline$\leq 0.70$ vs $>0.70 \times 10^{9} / \mathrm{L}$ & 2.38 (0.30 to 18.95$)$ & 0.413 & - & - \\
\hline \multicolumn{5}{|l|}{ NLR } \\
\hline$\geq 7.05$ vs $<7.05$ & $0.28(0.01$ to 5.48$)$ & 0.398 & - & - \\
\hline \multicolumn{5}{|l|}{ dNLR } \\
\hline$\geq 4.19$ vs $<4.19$ & 33.37 (1.56 to 714.58 ) & 0.025 & 18.99 (3.58 to 100.65$)$ & 0.001 \\
\hline COPD & 0.78 (0.09 to 6.38$)$ & 0.814 & - & - \\
\hline Dyspnea & 33.46 (3.71 to 301.73 ) & 0.002 & 20.38 (3.55 to 117.02$)$ & 0.001 \\
\hline Fatigue & 1.12 (0.18 to 7.18$)$ & 0.901 & - & - \\
\hline
\end{tabular}

The sample size for fitting the full model and simplified model was 156 .

COPD, chronic obstructive pulmonary disease; dNLR, derived NLR; NLR, neutrophil-to-lymphocyte ratio; WBC, peripheral white blood cell.

rate was higher for patients receiving immunotherapy and for those undergoing surgery. ${ }^{6}$ Other studies linked the risk of severe events with receipt of surgery or chemotherapy within 1 month, ${ }^{9}$ receipt of antitumor treatment within 14 days of COVID-19 diagnosis, ${ }^{12}$ receipt of chemotherapy within the prior 3 months, particularly among patients with active or metastatic cancer, ${ }^{22}$ and receipt of

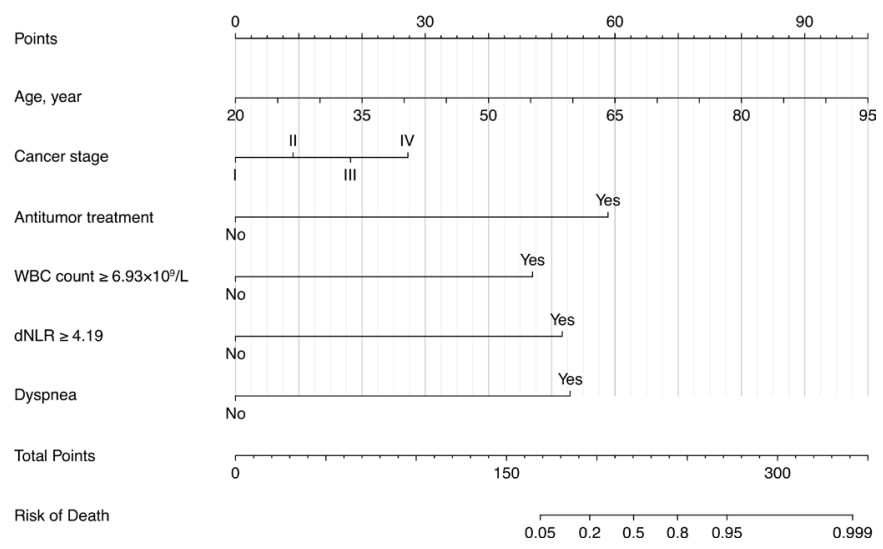

Figure 3 Nomogram for predicting mortality among patients with COVID-19 and solid tumors. dNLR, derived neutrophilto-lymphocyte ratio; WBC, peripheral white blood cell. chemotherapy within 4 weeks before symptom onset (the latter a multicenter study). ${ }^{13}$ Chemotherapy is well-known to deplete WBCs, particularly neutrophils, which has been linked with potentially lethal systemic infections. ${ }^{23}$ Immunotherapy with natural or engineered $\mathrm{T}$ cells could result in an unrestrained immune response, with ensuing deleterious inflammation. ${ }^{24}$ Given the particularly high mortality risk associated with receipt of antitumor treatment, presumably postponing surgery and immunotherapy and perhaps switching from cytotoxic chemotherapy to less toxic treatments during the COVID-19 pandemic may help to avert anticancer therapy-induced complications of COVID-19. ${ }^{25}$ Further studies of the effects of de-escalating antitumor regimens would also be informative for treatment of patients with COVID-19 and cancer.

A local inflammatory response can lead to systemic inflammation, which can contribute significantly to cancer progression. ${ }^{26}{ }^{27}$ WBCs have important roles in both immune and inflammatory processes, and dNLR is an indicator of systemic inflammation. The ability of WBC count and dNLR to predict prognosis for patients with solid tumors has been reported previously. ${ }^{28-31}$ In the present study, our nomogram included both WBC count 
and dNLR for predicting the probability of mortality in patients with COVID-19 with solid tumors. We found that the mortality risk increased for patients with high WBC counts and dNLR. This finding is consistent with those from another study in which elevated WBC count and dNLR were more common in patients with severe COVID-19 than in patients with less severe disease. ${ }^{32}$ Not surprisingly, the optimal cutoff value for dNLR for distinguishing non-survivors from survivors among patients with COVID-19 in our previous work (4.19) was larger than that for distinguishing severe from less severe illness (2.8). ${ }^{32}$

Dyspnea refers to a multidimensional sensation of increased respiratory effort, primarily manifested as labored breathing. Our results indicated that about $40 \%$ of all patients in this study presented with dyspnea and that dyspnea was more common among non-survivors $(70.3 \%)$ than among survivors $(33.0 \%)$. This disparity between non-survivors and survivors was in accordance with early studies comparing the frequency of dyspnea in patients with severe versus less severe COVID-19. 334 Despite the relatively high frequency observed in patients with severe illness (including death), the proportions of patients presenting with dyspnea have varied across studies. One reason for this variation was the difference in COVID-19 severity across studies. It is not surprising that patients who were treated in hospitals within COVID-19 epicenters would be much more seriously ill than patients located elsewhere, in part because of shortages in healthcare facilities and workers in the epicenters. Our nomogram illustrated that the mortality risk was higher in patients presenting with dyspnea on admission than those without dyspnea $(\mathrm{OR}=20.38)$. Another group also identified presenting with dyspnea as a risk factor for critical illness among patients hospitalized with COVID$19 .^{35}$ Logically, having information on the presence of this symptom at admission would help to improve the precision of nomograms for patients with COVID-19 who also have solid tumors.

Our study had some limitations. First, variations in the type of antitumor treatment could have distinct effects on prognosis for patients with COVID-19 and solid tumors; however, we did not consider the effects of antitumor treatments by type because the sample size of current study was insufficient for such assessment (online supplemental table S3). Second, although we collected data from 216 patients at 32 hospitals, only 37 patients had died at the time of analysis, and this number may be insufficient for multivariate logistic regression analysis with six independent variables, according to Harrell's guideline. ${ }^{36}$ The relatively small sample size may also explain the wide 95\% CIs around the ORs estimated from the multivariate logistic regression model. Nevertheless, the ORs for some variables were still statistically significant, suggesting that these variables could be important risk factors for mortality and would be valuable for predicting prognosis. Studies with larger numbers of patients may be better suited to explore other factors influencing prognosis for patients with COVID-19 and solid tumors and would further improve the predictive ability of the model in the future. Third, we did not perform external validation for the model fitted, since no data were available for such an assessment. However, we did attempt internal validation in an effort to prevent data overinterpretation, and the result suggested satisfactory interval validation of the model.

\section{CONCLUSION}

In conclusion, increasing age, receipt of antitumor treatment within 3 months of COVID-19 diagnosis, high WBC count and dNLR, and presenting with dyspnea on admission were independent predictors of mortality for patients with COVID-19 and solid tumors. The nomogram we developed can successfully predict mortality risk for individual patients. Future studies with larger numbers of patients will be useful for updating and validating this nomogram to improve predictions of in-hospital mortality among patients with COVID-19 and solid tumors.

\section{Author affiliations}

${ }^{1}$ Cancer Center, Renmin Hospital of Wuhan University, Wuhan, China 2Department of Radiation Oncology, Shandong Cancer Hospital and Institute, Shandong First Medical University and Shandong Academy of Medical Sciences, Jinan, China

${ }^{3}$ State Key Laboratory of Organ Failure Research, Department of Biostatistics, Guangdong Provincial Key Laboratory of Tropical Disease Research, School of Public Health, Southern Medical University, Guangzhou, China

${ }^{4}$ Department of Orthopaedic Surgery, Tongji Hospital, Tongji Medical College, Huazhong University of Science and Technology, Wuhan, China

${ }^{5}$ Department of Oncology, Tongji Hospital, Tongji Medical College, Huazhong University of Science and Technology, Wuhan, China

${ }^{6}$ Department of Oncology, The Fifth Hospital of Wuhan, Wuhan, China ${ }^{7}$ Cancer Center, Union Hospital, Tongji Medical College, Huazhong University of Science and Technology, Wuhan, China

${ }^{8}$ Department of Respiratory and Critical Care Medicine, Xiaogan Hospital Affiliated to Wuhan University of Science and Technology, Xiaogan, China

Acknowledgements We thank all following physicians for patient enrollment: Qibin Song (Renmin Hospital of Wuhan University), Yuan Chen (Tongji Hospital, Tongji Medical College, Huazhong University of Science and Technology), Qun Wang (The Fifth Hospital of Wuhan), Xiaorong Dong (Union Hospital, Tongji Medical College, Huazhong University of Science and Technology), Zhibin Xie (Xiaogan Hospital Affiliated to Wuhan University of Science and Technology), Linjun Li (Hubei Provincial Hospital of Integrated Chinese and Western Medicine), Weidong Hu (Zhongnan Hospital of Wuhan University), Zhiguo Rao (General Hospital of Central Theater Command, People's Liberation Army), Xiuli Luo (Hubei Provincial Hospital of TCM), Gang Feng (Wuhan Fourth Hospital [Puai Hospital], Tongji Medical College, Huazhong University of Science and Technology), Jing Liu (Huanggang Central Hospital), Xinhua Xu (Yichang Central People's Hospital), Ruizhi Ran (The Central Hospital of Enshi Tujia and Miao Autonomous Prefecture), Jiyuan Yang (First Affiliated Hospital of Yangtze University), Jun Jin (Ezhou Central Hospital), Zhiguo Luo (Taihe Hospital, Hubei University of Medicine), Hongda Lu (The Central Hospital of Wuhan, Tongji Medical College, Huazhong University of Science and Technology), Jie Ren (General Hospital of The Yangtze River Shipping), Li Kuang (Affiliated Dongfeng Hospital, Hubei University of Medicine), Runkun Wang (The first people's hospital of Guangshui), Yanhua Xu (Jingzhou Central Hospital), Youhong Dong (Xiangyang No.1 People's Hospital, Hubei University of Medicine), Zuowei Hu (Wuhan No. 1 Hospital), Chiding Hu (Affiliated Hospital of Jianghan University), Dongfeng Pan (Suizhou Hospital, Hubei University of Medicine), Gangsheng Wang (Huangshi Central Hospital of Edong Healthcare), Hanlin Wu (The First People's Hospital of Jingmen), Jianhai Sun (Hubei No. 3 People's Hospital), Liqiong Luo (Tianyou Hospital Affiliated to Wuhan University of Science and Technology), Mingyu 
Liu (The No. 9 hospital of Wuhan), Tienan Yi (Xiangyang Central Hospital, Hubei University of Medicine), Zhihong Zhang (Gong'an County People's Hospital).

Contributors $C-Q 0, Q H$ and $Q S$ conceived study. YY, HG, YC, QW, XD and $Z X$ collected data. Z-YZ analyzed data. C-Q0, QH and QS interpreted the results. CL, LL and KS wrote the first draft. All authors critically revised the draft and approved the final version.

Funding This work was supported by National Natural Science Foundation of China $(81973140,81670144)$.

\section{Competing interests None declared.}

Patient consent for publication Not required.

Ethics approval This study was approved by the Ethical Committee of Renmin Hospital of Wuhan University and all participating hospitals. Informed consent was waived for this study. Patient confidentiality was maintained throughout data collection and analysis.

Provenance and peer review Not commissioned; externally peer reviewed.

Data availability statement Data are available upon reasonable request. The datasets used and/or analyzed during the current study are available from the corresponding author upon reasonable request.

Open access This is an open access article distributed in accordance with the Creative Commons Attribution Non Commercial (CC BY-NC 4.0) license, which permits others to distribute, remix, adapt, build upon this work non-commercially, and license their derivative works on different terms, provided the original work is properly cited, appropriate credit is given, any changes made indicated, and the use is non-commercial. See http://creativecommons.org/licenses/by-nc/4.0/.

\section{ORCID iD}

Chun-Quan Ou http://orcid.org/0000-0001-6866-7213

\section{REFERENCES}

1 World Health Organization. WHO Director-General's opening remarks at the media briefing on COVID-19 - 11, 2020. Available: https://www.who.int/dg/speeches/detail/who-director-general-sopening-remarks-at-the-media-briefing-on-covid-19-11-march-2020 [Accessed 20 Jun 2020].

2 World Health Organization. Coronavirus disease (COVID-19) situation report - 151. Available: https://www.who.int/docs/default-source/ coronaviruse/situation-reports/20200619-covid-19-sitrep-151.pdf? sfvrsn=8b23b56e_2 [Accessed 20 Jun 2020]

3 World Health Organization. Cancer. Available: https://www.who.int/ news-room/fact-sheets/detail/cancer [Accessed 20 Jun 2020].

4 World Health Organization. Press release No. 263, latest global cancer data: cancer burden rises to 18.1 million new cases and 9.6 million cancer deaths in 2018. Available: https://www.who.int/cancer/ PRGlobocanFinal.pdf [Accessed 20 Jun 2020].

5 Kamboj M, Sepkowitz KA. Nosocomial infections in patients with cancer. Lancet Oncol 2009;10:589-97.

6 Dai M, Liu D, Liu M, et al. Patients with cancer appear more vulnerable to SARS-COV-2: a multicenter study during the COVID-19 outbreak. Cancer Discov 2020;10:783-91.

7 Guan W-J, Liang W-H, Zhao Y, et al. Comorbidity and its impact on 1590 patients with COVID-19 in China: a nationwide analysis. Eur Respir J 2020;55:2000547.

8 He W, Chen L, Chen L, et al. COVID-19 in persons with haematological cancers. Leukemia 2020;34:1637-45.

9 Liang W, Guan W, Chen R, et al. Cancer patients in SARSCoV-2 infection: a nationwide analysis in China. Lancet Oncol 2020;21:335-7.

10 Qin C, Zhou L, Hu Z, et al. Dysregulation of immune response in patients with coronavirus 2019 (COVID-19) in Wuhan, China. Clin Infect Dis 2020;71:ciaa248:762-8.

11 Chen N, Zhou M, Dong X, et al. Epidemiological and clinical characteristics of 99 cases of 2019 novel coronavirus pneumonia in Wuhan, China: a descriptive study. Lancet 2020;395:507-13.

12 Zhang L, Zhu F, Xie L, et al. Clinical characteristics of COVID-19infected cancer patients: a retrospective case study in three hospitals within Wuhan, China. Ann Oncol 2020;31:894-901.
13 Yang K, Sheng Y, Huang C, et al. Clinical characteristics, outcomes, and risk factors for mortality in patients with cancer and COVID-19 in Hubei, China: a multicentre, retrospective, cohort study. Lancet Oncol 2020;21:904-13.

14 lasonos A, Schrag D, Raj GV, et al. How to build and interpret a nomogram for cancer prognosis. J Clin Oncol 2008;26:1364-70.

15 National Health Commission of the People's Republic of China. Diagnosis and treatment protocols of pneumonia caused by a novel coronavirus (trial version 7) [in Chinese]. Available: http://www.nhc. gov.cn/yzygj/s7653p/202003/46c9294a7dfe4cef80dc7f5912eb1989/ files/ce3e6945832a438eaae415350a8ce964.pdf [Accessed 20 Jun 2020].

16 Snee RD. Some aspects of nonorthogonal data analysis, part 1 developing prediction equations. Journal of Quality Technology 1973:5:67-79.

17 Moons KGM, Altman DG, Reitsma JB, et al. Transparent reporting of a multivariable prediction model for individual prognosis or diagnosis (TRIPOD): explanation and elaboration. Ann Intern Med 2015;162:W1-73

18 Balachandran VP, Gonen M, Smith JJ, et al. Nomograms in oncology: more than meets the eye. Lancet Oncol 2015;16:e173-80.

19 DeLong ER, DeLong DM, Clarke-Pearson DL. Comparing the areas under two or more correlated receiver operating characteristic curves: a nonparametric approach. Biometrics 1988;44:837-45.

20 Hosmer DW, Lemeshow S. Applied logistic regression. New York, USA: John Wiley and Sons, 2000.

21 Mehta V, Goel S, Kabarriti R, et al. Case fatality rate of cancer patients with COVID-19 in a New York hospital system. Cancer Discov 2020;10:935-41.

22 American Association for Cancer Research. AACR virtual annual meeting I: a global perspective of COVID-19 in patients with cancer. Available: https://www.aacr.org/professionals/blog/aacr-virtualannual-meeting-i-a-global-perspective-of-covid-19-in-patients-withcancer/ [Accessed 20 Jun 2020].

23 Gupta A, Abbasi B, Gupta S. Management of chemotherapy induced neutropenia - an unmet clinical need. Am J Biomed Sci Res 2019;4:313-8.

24 Cancer Health. Cancer patients with COVID-19 may have higher risk of severe illness and death. Available: https://www.cancerhealth. com/article/cancer-patients-covid19-may-higher-risk-severe-illnessdeath [Accessed 20 Jun 2020].

25 van de Haar J, Hoes LR, Coles CE, et al. Caring for patients with cancer in the COVID-19 era. Nat Med 2020;26:665-71.

26 Singh R, Mishra MK, Aggarwal H. Inflammation, immunity, and cancer. Mediators Inflamm 2017;2017:6027305.

27 Aggarwal BB, Vijayalekshmi RV, Sung B. Targeting inflammatory pathways for prevention and therapy of cancer: short-term Friend, long-term foe. Clin Cancer Res 2009;15:425-30.

28 Proctor MJ, McMillan DC, Morrison DS, et al. A derived neutrophil to lymphocyte ratio predicts survival in patients with cancer. $\mathrm{Br} J$ Cancer 2012;107:695-9.

29 Su S, Liu L, Li C, et al. Prognostic role of pretreatment derived neutrophil to lymphocyte ratio in urological cancers: a systematic review and meta-analysis. Int J Surg 2019;72:146-53.

30 Duan J, Pan L, Yang M. Preoperative elevated neutrophil-tolymphocyte ratio (NLR) and derived NLR are associated with poor prognosis in patients with breast cancer: a meta-analysis. Medicine 2018;97:e13340.

31 Wang Y-L, Ge X-X, Wang Y, et al. The values of applying classification and counts of white blood cells to the prognostic evaluation of resectable gastric cancers. BMC Gastroenterol 2018;18:99

32 Yang A-P, Liu J-P, Tao W-Q, et al. The diagnostic and predictive role of NLR, d-NLR and PLR in COVID-19 patients. Int Immunopharmacol 2020;84:106504.

33 Huang C, Wang Y, Li X, et al. Clinical features of patients infected with 2019 novel coronavirus in Wuhan, China. Lancet 2020;395:497-506.

34 Guan W-J, Ni Z-Y, Hu Y, et al. Clinical characteristics of coronavirus disease 2019 in China. N Engl J Med 2020;382:1708-20.

35 Liang W, Liang H, Ou L, et al. Development and validation of a clinical risk score to predict the occurrence of critical illness in hospitalized patients with COVID-19. JAMA Intern Med 2020:e202033.

36 Harrell FJ. Regression modeling strategies with applications to linear models, logistic regression, and survival analysis. New York, NY: Springer Verlag, 2001 